\title{
Effects of Electroacupuncture Stimulation at Frequencies Near the Heart Rate on the Microcirculatory Blood Flow
}

\author{
Fong-Cheng Lin ${ }^{1}$, Hsin Hsiu ${ }^{2,}$, Yao-Chun Lin ${ }^{3}$, Ching-Yi Chung ${ }^{4}$, Wei-Chen Hsu ${ }^{4}$, Chao-Tsung Chen ${ }^{4}$ \\ ${ }^{1}$ Department of Rehabilitation, Taipei City Hospital RenAi Branch, Taiwan \\ ${ }^{2}$ Graduate Institute of Biomedical Engineering, National Taiwan University of Science and Technology, Taiwan \\ ${ }^{3}$ Department of Electrical Engineering, Yuan Ze University, Taiwan \\ ${ }^{4}$ Department of Traditional Chinese Medicine, Taipei City Hospital RenAi Branch, Taiwan
}

Copyright $(\mathcal{C} 2015$ by authors, all rights reserved. Authors agree that this article remains permanently open access under the terms of the Creative Commons Attribution License 4.0 International License

\begin{abstract}
Motivation: The frequency of electroacupuncture (EA) could substantially influence the induced response. We compared the effects of EA at frequencies at around the heart rate (HR) and $50 \%$-higher-than-HR frequency on the microcirculatory blood flow (MBF). Methods: Skin-surface laser Doppler measurements were performed in healthy volunteers in three groups: (1) Group A ( $\mathrm{n}=14)$, premeasurement-HR-frequency EA was applied; and (2) Group B $(\mathrm{n}=13)$, 50\%-higher-than-HR-frequency EA. (3) Group C $(n=16)$, no EA was applied. Each experiment involved recording a 20-min baseline-data sequence followed by an effect-data sequence obtained at $0-20 \mathrm{~min}$ after stopping $20 \mathrm{~min}$ of 0.08-mA EA. Results: Beat-to-beat time-domain waveform analysis (including the pulse width, flow rise time, and microcirculatory blood-flow variability) revealed that following EA, the MBF supply at the stimulated site was improved in both groups, and that the MBF perfusion through arteriolar openings appeared to be more efficient in Group A than in Group B. Conclusion: The obtained data may help to establish a new noninvasive method for studying the mechanisms underlying the MBF response induced by EA.
\end{abstract}

Keywords Electro-acupuncture, Laser Doppler, Microcirculatory Blood Flow Variability

\section{Introduction}

The ancient therapeutic technique of acupuncture and its modern modification, electro-acupuncture (EA), have been shown to be effective in several pathological conditions. The physiological mechanisms underlying EA have been widely studied, as has its effects on the microcirculatory blood flow (MBF), such as that on cardiovascular remodeling and nitric oxide in spontaneously hypertensive rats [1]. Moreover, EA treatment has been noted to be useful in improving ovarian blood flow [2], and in protecting the brain by increasing MBF to the ischemic region via neural regulation [3].

Among the various factors that can be manipulated when performing the EA procedure, the effects of varying the EA frequency have been widely studied. For example, 2-Hz EA induced a beneficial neuroendocrine effect in rats with polycystic ovary syndrome [4], and was useful as a supportive therapy to reduce nerve-growth-factor-induced side effects in adult healthy rats [5]. It was also found in rats with steroid-induced polycystic ovaries that the increase in the ovarian MBF was larger for $2-\mathrm{Hz}$ than for an $80-\mathrm{Hz}$ EA stimulation [2]. These results indicate that the EA frequency can substantially influence the induced responses, and thus understanding the underlying mechanisms might allow the treatment efficacy of EA to be improved.

Laser Doppler Flowmetry (LDF) is a widely used technique for MBF monitoring due to its advantages of a good frequency response, ease of application, and tissue specificity, and is therefore well suited for noninvasive investigations of the microvascular responses induced by various forms of treatment or stimulation [6-8]. We have previously noted that acupuncture can increase the MBF by reducing the perfusion resistance at the needled site $[6,9,10]$. The local responses induced by EA, such as the contraction of arterial smooth muscle or even the skeletal muscle, vary with its magnitude. Since the contraction of arterial smooth muscle might provide a force that augments the effects of the heartbeat on the terminal MBF supply, whether or not the EA frequency is near to the heart rate (HR) might affect the induced MBF response. Although many studies have explored the effects of the EA frequency, few of them have focused on frequencies near to the HR.

MBF can be pushed through the arterial openings (AO) by the propelling force of the pulse blood pressure generated from the heartbeat and transmitted through the artery. When current passes through local tissue near the vessel, it is possible that changes in the vessel wall can be induced, and 
hence change the condition for the blood to pass through AO. Therefore it is possible that deviation for the electrical stimulating frequency from the HR can also affect local condition of MBF perfusion. In the present study we carried out skin-surface LDF measurements around the EA site and time-domain waveform analysis in order to compare the frequency-specific MBF effects of EA at frequencies around and $50 \%$ higher than the HR. The data thus obtained might aid the development of objective methods for studying the underlying mechanisms and identifying the operating conditions of EA.

\section{Materials and Methods}

\subsection{Experimental Setup and Data Acquisition}

Experiments were carried out on healthy male volunteers without signs or symptoms of cardiovascular or neurological disease. The subjects were all Taiwan natives, were lightly clothed, supine, and were allowed to stabilize for at least 20 min before commencing recording. The environmental temperature was within $23-25^{\circ} \mathrm{C}$ during the entire measuring period. The institutional ethics committee at Taipei City Hospital approved the study protocol, and each volunteer gave informed consent before entering the study. Tea, coffee, alcohol, and smoking were forbidden on the day before experiments. All subjects did not exercise or consume food for at least 1 hour before each experiment $[6,9,10]$.

The ECG and LDF signals were measured simultaneously and noninvasively (Fig.1). ECG signals were measured by surface electrodes, and acquired by a preamplifier (lead II, RA-LL; 6600-series, Gould, USA). LDF (VP1 probe; MBF3, Moor Instruments, UK) was used to measure the microcirculatory flux with a time constant of $0.001 \mathrm{~s}$, a cut-off frequency of $14.9 \mathrm{kHz}$ and a sampling frequency of $40 \mathrm{~Hz}$. The laser operating wavelength and output power were $780 \mathrm{~nm}$ and less than $1.6 \mathrm{~mW}$, respectively. Subjects were asked to relax and breathe naturally throughout the measurement period so as to avoid motion artifacts. The signals were connected to an analog-to-digital converter card (PCI-9111DG, Adlink Technology, Taiwan) operating at a sampling rate of $1024 \mathrm{~Hz}[6,9,10]$.

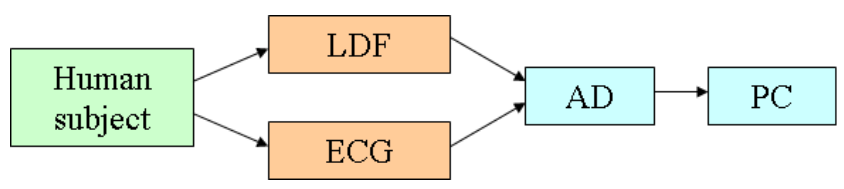

Figure 1. Experimental setup

The measuring sites of the LDF probe (see Fig. 2a) were at left Hegu (LI-4; Site 1), an acupoint located between the thumb and the index finger on the back of the hand, and a nearby nonacupoint (Site 2) that was halfway between Hegu and Yang-Tsih (LI-5). The LDF probe was held vertically onto the skin surface by a holder with a radius of around 6 $\mathrm{mm}$. During the measurement, the subject was supine on a measurement couch. Each assessment involved making the following recordings: the 20-min baseline (M0) data were recorded prior to EA, after which the acupuncture needle (stainless steel needle, gauge \#30; Chianhuei, Taiwan) was inserted perpendicularly into the left Hegu ( $2.5 \mathrm{~cm}$ in length) and Quchi (LI-11; $5.0 \mathrm{~cm}$ in length) acupoints to depths of around 1.2 and $2.5 \mathrm{~cm}$, respectively. The needles at each site were connected to an electrical stimulator (D0310K, Ching-Ming, Taiwan) and were electrically stimulated with square-wave pulses (200-ìs duration) with alternating polarity (see Fig. 2b). The needle was then withdrawn to stop EA, with another $20 \mathrm{~min}$ of data then being recorded (M1). Similar with the method used in several of our previous works $[6,9]$, we used a ball-point pen to sketch along the margin of the probe holder on the skin surface to help to ensure the reproducibility of the location for the LDF measurement sites.

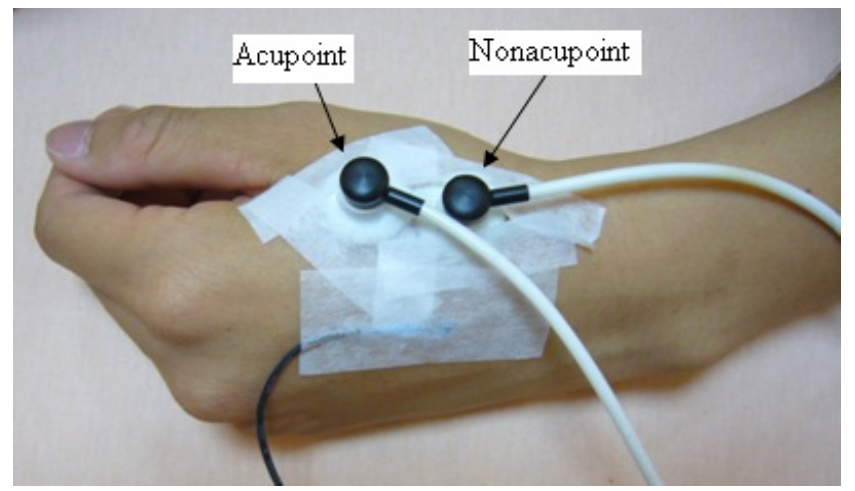

(a)

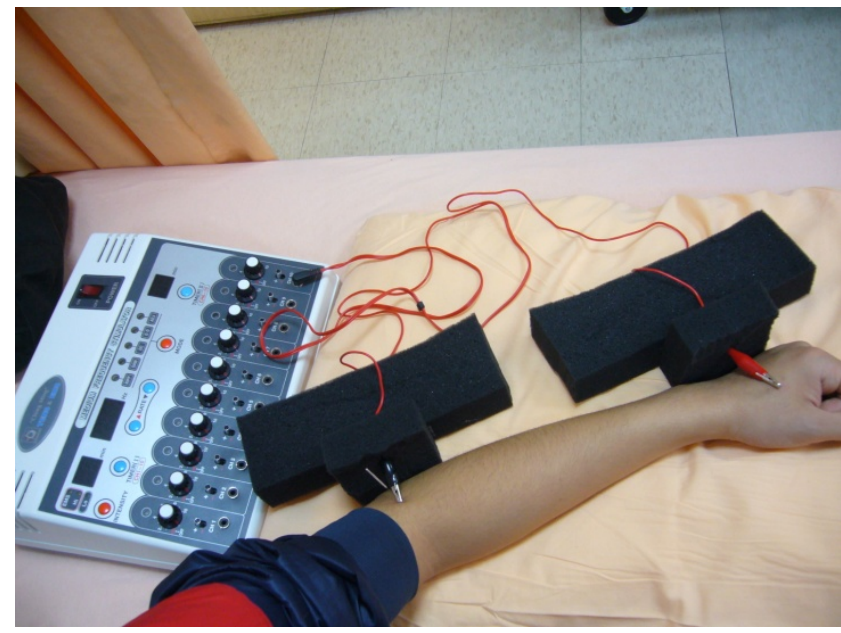

(b)

Figure 2. Photographs of the experimental setup, showing the locations of the measurement (a) and needled (b) sites.

The effects of different stimulation frequencies were compared between the following groups: (1) Group A ( $\mathrm{n}=$ 14 , aged $31.0 \pm 14.4$ years), in which EA was applied at the frequency of the average premeasurement $\mathrm{HR}$ of each subject; (2) Group B ( $\mathrm{n}=13$, aged $28.7 \pm 13.0$ years), in which EA was applied at a frequency that was $50 \%$ higher than the HR; and (3) Control Group C ( $\mathrm{n}=16$, aged $27.8 \pm$ 
10.6 years), in which no EA was applied. Weak (0.08-mA) EA was applied for $20 \mathrm{~min}$; such a low amplitude was used in order to minimize the possible interference effects of other physiological or sensory pathways.

Before the baseline and after the M1 period, fundamental physiological parameters of the subject, including the heart rate, systolic and diastolic blood pressures were measured by a sphygmomanometer (HEM-722C, Omron). A thermistor was attached to the skin surface to monitor the skin surface temperature near Site 1 . The resistance of the thermistor was transformed into voltage using a custom-made circuit, which was also sampled by the analog-to-digital converter card. The temperature stability during the baseline period was considered acceptable when the variation was less than $1.0^{\circ} \mathrm{C}[10]$.

Table 1. Changes in fundamental physiological parameters and the monitored skin temperature.

\begin{tabular}{|c|c|c|c|c|c|c|c|}
\hline & & $\begin{array}{c}\text { Group } \\
\text { A }\end{array}$ & & $\begin{array}{c}\text { Group } \\
\text { B }\end{array}$ & & $\begin{array}{c}\text { Group } \\
\text { C }\end{array}$ & \\
\hline & & mean & SD & mean & SD & mean & SD \\
\hline HR & M0 & 69.21 & 7.68 & 71.62 & 9.21 & 70.3 & 9.92 \\
\hline (beats/min) & M1 & $64.93^{*}$ & 9.47 & $67.31^{*}$ & 8.23 & 68.6 & 8.79 \\
\hline SBP & M0 & 111.2 & 15.2 & 118.5 & 12.8 & 117.3 & 13.6 \\
\hline$(\mathrm{mm}-\mathrm{Hg})$ & M1 & 111.2 & 17.7 & 114.1 & 18.0 & 115.2 & 18.6 \\
\hline DBP & M0 & 68.1 & 7.1 & 71.1 & 8.7 & 71.7 & 9.92 \\
\hline$(\mathrm{mm}-\mathrm{Hg})$ & M1 & $72.4 *$ & 11.5 & 73.7 & 10.4 & 74.4 & 11.8 \\
\hline Temperature & M0 & 31.6 & 2.2 & 32.2 & 2.1 & 32.1 & 2.4 \\
\hline$\left({ }^{\circ} \mathrm{C}\right)$ & M1 & 30.5 & 2.7 & 31.0 & 2.4 & 30.9 & 2.7 \\
\hline
\end{tabular}

The values were measured before M0 and after M1. “*” indicates $\mathrm{p}<0.05$ compared with the baseline value by two-tailed paired t-test.

\subsection{Signal Analysis}

Data files containing spikes that were too large relative to the mean flux value were discarded, since this implied the presence of motion artifacts. Several parameters of the LDF signal were calculated to elucidate the microcirculatory effects induced by EA (Fig.3). The DC component of the flux) (DCflux) was defined as the mean value of the LDF signals during each 20-min measurement period. In determining the parameter of the pulsatile LDF waveform, the LDF flux signal was filtered by an 11th-order Chebyshev digital high-pass filter with a cut-off frequency of $0.01 \mathrm{~Hz}$ to eliminate the baseline drift. The two neighboring $\mathrm{R}$ peaks of the ECG signal were used to help to identify the cut-off points of each pulse for LDF signals to define each "pulse" of the MBF. The cut-off point was defined as the minimum LDF flux signal within a 300-ms search window located after the corresponding ECG R-peak (Fig.4). For each pulse, the mean component was defined as the average value and the pulse component as the difference between the maximum and minimum of the LDF signal. During each 20-min measurement period, the AC component of the flux (ACflux) was defined as the mean value of the LDF pulse component, the ADflux defined as the mean value of the ratio between the LDF pulse component and the mean component (i.e., the ACflux to DCflux ratio), the flow rise time (FRT) defined as the average time interval between the foot point and the peak point of the LDF signal, and the pulse width (PW) defined as the average time interval between the $80 \%$ rising and falling points (i.e., the points at which the flux was $80 \%$ of the pulse peak on the rising and falling edges, respectively)7,9. The MBFV index, defined as the coefficient of variation, DCCV (mean $\pm \mathrm{SD}$ ) of the mean component of all the pulses during the 20-min period [11, 12], could then be calculated.

All signal processing was performed with MATLAB (MathWorks, Natick, MA, USA). All statistical analyses were carried out using SPSS, version 13.0. The responses in the fundamental physiological and LDF parameters were tested with one-way repeat-measures ANOVA. The Bonferroni method was used for post-hoc analysis. The level of significance was defined as $\mathrm{p}<0.05$; all $\mathrm{p}$-values were two-sided hypotheses.

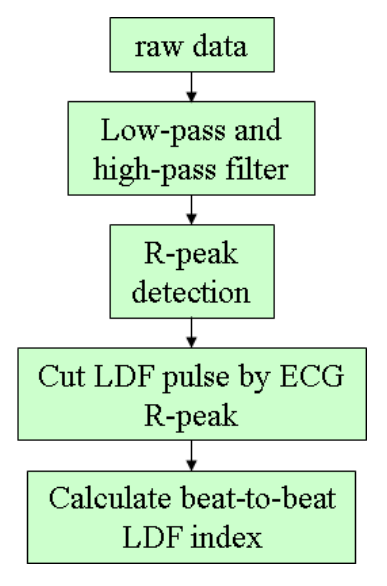

Figure 3. Procedure of data analysis 


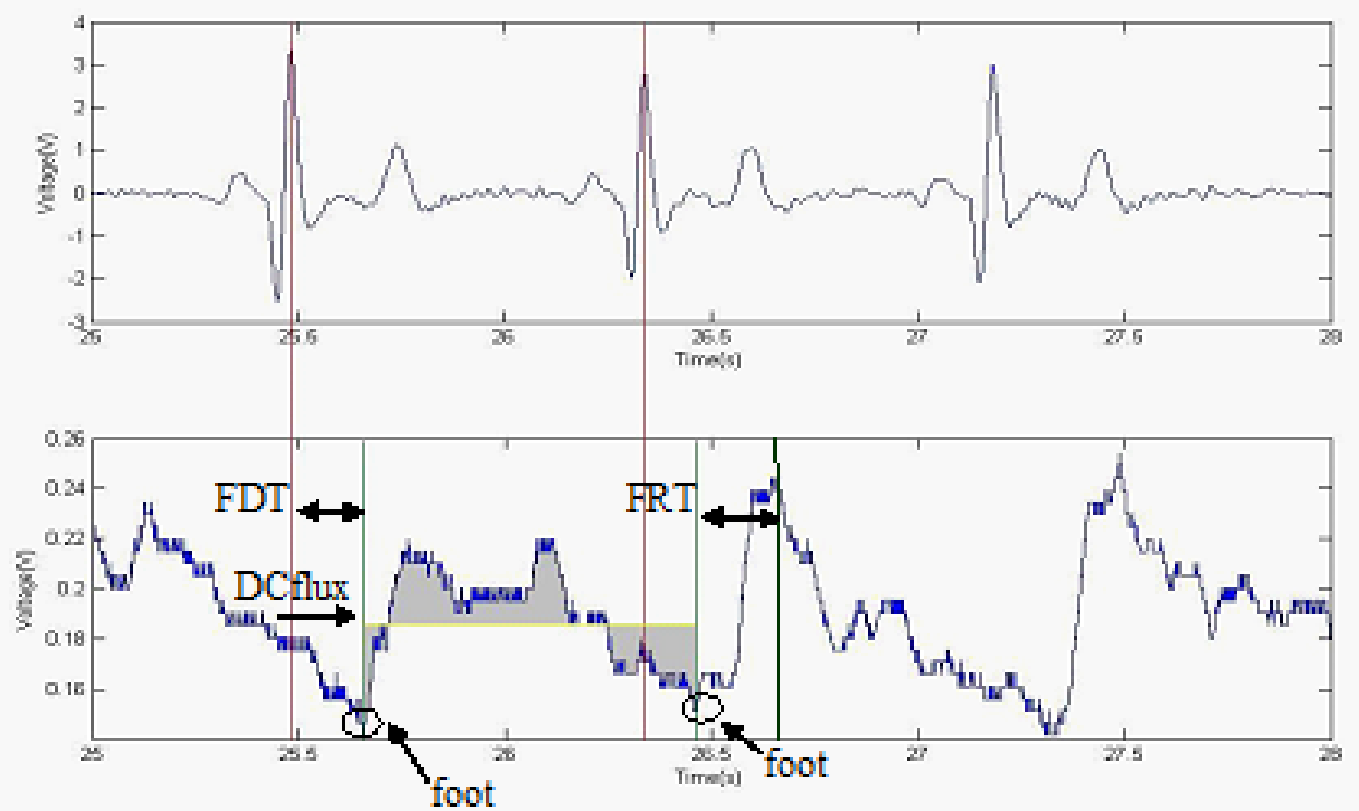

Figure 4. Typical measured waveforms and definition of beat-to-beat LDF parameters. Upper: ECG; lower: LDF signal.

Table 2. Changes in LDF parameters following EA.

\begin{tabular}{|c|c|c|c|c|c|c|c|c|c|}
\hline & & & Group A & & Group B & & Group C & & $\begin{array}{c}\text { Between- } \\
\text { group comparison }\end{array}$ \\
\hline & & & mean & SD & mean & SD & mean & $\mathrm{SD}$ & \\
\hline \multirow[t]{4}{*}{ DCflux } & Site 1 & M0 & 0.230 & 0.063 & 0.315 & 0.138 & 0.266 & 0.117 & $\mathrm{AB}^{*}$ \\
\hline & & M1 & $0.347 *$ & 0.195 & $0.501 *$ & 0.337 & 0.263 & 0.106 & $\mathrm{AB}+, \mathrm{BC}^{*}$ \\
\hline & Site 2 & M0 & 0.250 & 0.096 & 0.309 & 0.189 & 0.234 & 0.110 & \\
\hline & & M1 & 0.249 & 0.137 & 0.282 & 0.211 & 0.220 & 0.090 & \\
\hline \multirow[t]{4}{*}{ ACflux } & Site 1 & M0 & 0.015 & 0.010 & 0.025 & 0.020 & 0.020 & 0.010 & \\
\hline & & M1 & $0.031 *$ & 0.026 & $0.042+$ & 0.033 & 0.019 & 0.010 & $\mathrm{BC}^{*}$ \\
\hline & Site 2 & M0 & 0.026 & 0.017 & 0.033 & 0.026 & 0.021 & 0.012 & $\mathrm{BC}^{*}$ \\
\hline & & M1 & 0.026 & 0.020 & 0.032 & 0.037 & 0.020 & 0.011 & $\mathrm{BC}+$ \\
\hline \multirow[t]{4}{*}{ ADflux } & Site 1 & M0 & 0.065 & 0.032 & 0.066 & 0.037 & 0.076 & 0.035 & \\
\hline & & M1 & $0.081 *$ & 0.029 & 0.071 & 0.027 & 0.076 & 0.033 & \\
\hline & Site 2 & M0 & 0.096 & 0.032 & 0.090 & 0.037 & 0.090 & 0.033 & \\
\hline & & M1 & 0.105 & 0.064 & 0.098 & 0.041 & 0.088 & 0.031 & $\mathrm{AC}^{*}$ \\
\hline \multirow[t]{4}{*}{ PW } & Site 1 & M0 & 0.072 & 0.055 & 0.131 & 0.088 & 0.162 & 0.032 & $\mathrm{AC}^{*}$ \\
\hline & & M1 & $0.138 *$ & 0.113 & $0.200^{*}$ & 0.120 & 0.161 & 0.029 & \\
\hline & Site 2 & M0 & 0.137 & 0.080 & 0.167 & 0.100 & 0.161 & 0.033 & \\
\hline & & M1 & 0.115 & 0.059 & 0.156 & 0.084 & 0.160 & 0.032 & \\
\hline \multirow[t]{4}{*}{ FRT } & Site 1 & M0 & 0.228 & 0.041 & 0.204 & 0.071 & 0.223 & 0.040 & \\
\hline & & M1 & $0.207 *$ & 0.027 & 0.198 & 0.059 & 0.220 & 0.040 & \\
\hline & Site 2 & M0 & 0.213 & 0.066 & 0.177 & 0.053 & 0.200 & 0.025 & \\
\hline & & M1 & $0.181+$ & 0.023 & 0.176 & 0.057 & 0.203 & 0.035 & \\
\hline \multirow[t]{4}{*}{ DCCV } & Site 1 & M0 & 0.180 & 0.090 & 0.197 & 0.111 & 0.190 & 0.072 & \\
\hline & & M1 & $0.257 *$ & 0.116 & 0.230 & 0.182 & 0.192 & 0.068 & $\mathrm{AC}^{*}$ \\
\hline & Site 2 & M0 & 0.204 & 0.099 & 0.302 & 0.227 & 0.216 & 0.065 & $\mathrm{AB}^{+}$ \\
\hline & & M1 & $0.314^{*}$ & 0.256 & 0.286 & 0.326 & 0.221 & 0.063 & $\mathrm{AC}^{*}$ \\
\hline
\end{tabular}

PW and FRT are in seconds. "*” and "+” indicate $\mathrm{p}<0.05$ and $\mathrm{p}<0.1$, respectively, compared with the baseline value. There were no significant changes in Group C and at Site 2. 


\section{Results}

Among the basic physiological parameters listed in Table 1, HR significantly decreased in Groups A (EA was applied at the frequency of the average premeasurement HR of each subject) and B (EA was applied at a frequency that was $50 \%$ higher than the HR), and DBP significantly increased only in Group A. There were no significant changes in the control Group C (without applying any stimulation).

Table 2 reveals that compared to the baseline values, DCflux at the acupoint (Site 1) significantly increased in both EA groups (Groups A and B), while ACflux and ADflux significantly increased only at the acupoint in Group A.

For parameters of the LDF time-domain waveform, there were no significant changes at Site 2 in all groups and at Site 1 in Group C. At Site 1. the pulse width (PW) significantly increased in both EA groups (Groups A and B), whereas FRT significantly shortened only in Group A. For the microcirculatory blood flow variability (MBFV) parameter, the coefficient of variation of the mean component of all the pulses (DCCV) significantly increased at both sites in Group $\mathrm{A}$, whereas there were no significant changes in the other groups (Groups B and C).

\section{Discussion}

\subsection{MBF-supply Parameters}

This study analyzed the effects of EA on skin blood flow. At the EA site, DCflux significantly increased in both EA groups, and ACflux significantly increased only in Group A. There were no significant changes in DCflux and ACflux in Group C. This indicates that the MBF supply was improved at the stimulated site following the EA, despite it having a low amplitude of around $0.08 \mathrm{~mA}$.

One major drawback that limits the application of the LDF technique is that it provides only a relative qualitative measurement index of the local MBF supply. Therefore, when evaluating the MBF-supply condition, the present analysis also calculated the ACflux-to-DCflux ratio (ADflux), which could help to eliminate the interference effects on DCflux induced by variations between different individuals. We found that the ADflux increased only at the EA site in Group A. This indicates that a greater pulsatile portion of the MBF passed through the AO under a constant mean MBF supply. This implies that the proportion of the local MBF passing through AO could be increased by applying EA near to the HR (i.e., in Group A).

\subsection{Time-domain LDF Waveform Analysis}

Since the pulsatile portion of the BP is about $40 \mathrm{~mm}-\mathrm{Hg}$, about $1 / 3$ of the total $\mathrm{BP}$, the transverse wave can be an important wave mode in the arteries. When the wave is carried by the blood in the arteries, viscous effects of the blood would be large. In contrast, a transverse pulse wave carried by the propagating oscillation of the elastic walls is subject to a much lower attenuation [13]. At the end of the arterial system, the arterial wall becomes stiffer. It is possible that the beat-to-beat propelling force generated by the heartbeat can help to drive the blood through the highly resistive AO into the tissue [10]. Therefore the pulsatile LDF waveform may provide a tool for analyzing the blood-flow conditions at local vascular beds induced by various types of stimulation [7, 10]. Among the present analyzed time-domain waveform indexes, $\mathrm{PW}$ represents the injection period via $\mathrm{AO}$ of the peripheral vascular beds during beat-to-beat MBF perfusion [10, 12]. An increased PW for each pulse might therefore be associated with the longer injection period of AO. In the present study, PW significantly increased in both EA groups. This indicates that the improved DCflux supply might be attributable to the lengthened injection period and the resulting improved perfusion condition through local AO in these groups.

The flow rise time (FRT) represents the time required for each LDF pulse to rise from the foot point to the peak point, and hence can be used to evaluate the resistance experienced by blood passing through $\mathrm{AO}$. We found a significant decrease in FRT only in Group A, which indicates that EA can improve the perfusion resistance.

It is also noteworthy that the increase in the $\mathrm{M} 1 / \mathrm{M} 0$ ratio of PW was slightly larger in Group A (1.92) than in Group B (1.52). This further supports that the present low-frequency EA can improve the local MBF supply, and also that the perfusion conditions might have differed between Groups A and $\mathrm{B}$.

While DCflux was significantly improved in both groups, ACflux and ADflux significantly improved only in Group A. This indicates that the MBF perfusion through the AO might be higher when the EA frequency is matched to the HR (i.e., as in Group A). Applying EA to local tissue can induce the contraction of arterial smooth muscle, and might augment the force exerted by the heartbeat on the terminal MBF supply. This difference could be partly attributed to the contraction force induced by EA at a frequency near to the HR acting to assist the propelling force of the heartbeat transmitted along the artery that pushes the MBF through the AO into the local tissue. This could have been responsible for the lengthened PW and the shortened FRT in Group A. In contrast, the large discrepancy between the EA frequency and the HR in Group B would result in these two forces not acting together to push the MBF, and therefore those LDF parameters associated with the condition of the AO did not show the prominent improvement seen in Group A.

Changes in the fundamental physiological parameters provide further support for the above conjecture. The HR decreased significantly in both EA groups, with the decrease being slightly larger in Group A than in Group B. This indicates a slight decrease in the loading on the heart in Group A, which could increase the efficacy of the force provided by the heartbeat. Moreover, there were no significant changes in the systolic blood pressure, and the 
diastolic blood pressure only significantly increased in Group A. Since this implies a more efficient charging condition to the arterial compliance, the present results indicate that the improvement of the cardiac load was greater in Group A than in Group B, which also concurs with the above conjecture about the difference in the induced MBF responses between the two EA groups.

\subsection{MBFV (Microcirculatory Blood Flow Variability)}

Indexes of the beat-to-beat cardiovascular variability, such as the variability of the HR and of the blood-pressure, have been widely investigated for monitoring the activity of autonomic nervous modulation $[14,15]$. HR variability has been suggested to reflect the complexity of the heartbeat and cardiovascular adaptability in searching for optimal working conditions, and is therefore related to cardiovascular adjustments induced by various types of stimulation [16]. At the microcirculatory level, changes in MBFV parameters can be attributed to various microcirculatory regulatory mechanisms acting on the on-off tuning activities of $\mathrm{AO}$, and these might be useful for evaluating cardiovascular regulatory activity. For example, applying local-heating stimulation to humans and administering angiotensin II to rats has revealed significant correlations between changes in MBF supply and the beat-to-beat MBFV parameters, which suggests a connection between such parameters and the terminal MBF supply $[11,12]$. Beat-to-beat MBFV analysis could therefore be useful when developing and evaluating treatment techniques aimed at improving microcirculatory perfusion.

The present study found significant increases in MBFV only at both sites in Group A, which indicates that the applied EA prominently affected the regulatory activities (and thus DCCV of the local vascular beds) near to the stimulated site. The differences in the effects induced by different EA frequencies could be related to the vibration properties of the arterial wall. It was previously hypothesized that the radial forces acting on the elastic wall of the main artery are associated with filtering in the frequency domain, with a natural frequency occurring near to the $\operatorname{HR}[7,13,17]$. When the heart beats at near to the average HR in normal physiological condition (which was referred as the "frequency-matching" condition), the oscillation of the artery will be larger, which will in turn produce a larger pressure pulse and thereby improve the transmission of blood through $\mathrm{AO}$.

It is possible that the rhythm of the vascular smooth-muscle contraction induced by the applied EA was near to the HR in Group A. The presence of two forces pushing the $\mathrm{MBF}$ - the heartbeat and the external EA stimulation-might result in the local microcirculatory regulation utilizing the external EA to assist the heartbeat in distributing the blood supply into local vascular beds around the site of EA application. In contrast, in Group B, where the EA was applied at a frequency far from the HR, it would be more difficult for the local microcirculatory regulation to utilize the EA, resulting in no significant changes occurring in DCCV.

\section{Conclusions}

EA with near-HR frequency applied on the local skin improves the MBF supply at the stimulated site. Making measurements on the skin surface around the EA site could help to establish a noninvasive method for studying the mechanisms underlying the MBF response induced by EA.

\section{Acknowledgements}

The authors would like to thank the National Science Council for partial support of this work.

\section{REFERENCES}

[1] X. Xiong, C. You, Q. C. Feng, T. Yin, Z. B. Chen, P. Ball, L. $\mathrm{X}$. Wang. Pulse width modulation electro-acupuncture on cardiovascular remodeling and plasma nitric oxide in spontaneously hypertensive rats. Evidence-Based Complementary and Alternative Medicine, 2011:812160.

[2] E. Stener-Victorin, R. Kobayashi, O. Watanabe, T. Lundeberg, M. Kurosawa. Effect of electro-acupuncture stimulation of different frequencies and intensities on ovarian blood flow in anaesthetized rats with steroid-induced polycystic ovaries. Reproductive Biology and Endocrinology, Vol.2, pp.16, 2004.

[3] F. Zhou, J. Guo, J. Cheng, G. Wu, Y. Xia. Electroacupuncture increased cerebral blood flow and reduced ischemic brain injury: Dependence on stimulation intensity and frequency. Journal of Applied Physiology, Vol, No.6, pp.1877-1887, 2011.

[4] Y. Feng, J. Johansson, R. Shao, L. Mannerås, J. Fernandez-Rodriguez, H. Billig, E. Stener-Victorin. Hypothalamic neuroendocrine functions in rats with dihydrotestosterone-induced polycystic ovary syndrome: effects of low-frequency electro-acupuncture. PLoS One, Vol.4, No.8, pp.e6638, 2009.

[5] L. Aloe, L. Manni. Low-frequency electro-acupuncture reduces the nociceptive response and the pain mediator enhancement induced by nerve growth factor. Neuroscience Letter, Vol.449, No.3, pp.173-177, 2009.

[6] H. Hsiu, W.C. Hsu, C.L. Hsu, S.M. Huang. Assessing the effects of acupuncture by comparing needling the Hegu acupoint and needling nearby nonacupoints by spectral analysis of microcirculatory laser Doppler signals. Evidence-Based Complementary and Alternative Medicine, 2011:435928, 2011.

[7] H. Hsiu, W.C. Hsu, C.L. Hsu, W.C. Lin, M.H. Ho, C.T. Chen, H.F. Hu. Acute microcirculatory responses induced by skin-surface vibration stimulation at a frequency near the heart rate. Biorheology, Vol.49, pp.15-25, 2012.

[8] M. Jünger, H. Haase, L. Schwenke, J. Bichel, J. Schuren, A. 
Ladwig. Macro- and microperfusion during application of a new compression system, designed for patients with leg ulcer and concomitant peripheral arterial occlusive disease. Clinical Hemorheology and Microcirculation, Vol.53, pp.281-293, 2013.

[9] H. Hsiu, S.M. Huang, C.T. Chen, C.L. Hsu, W.C. Hsu. Acupuncture stimulation causes bilaterally different microcirculatory effects in stroke patients. Microvascular Research, Vol.81, pp.289-294, 2011.

[10] H. Hsiu, S.M. Huang, C.T. Chen, W.C. Hsu, F.C. Lin. Differences in the beat-to-beat parameters of skin-surface pulsatile laser-Doppler waveforms between stroke and normal subjects, Clinical Hemorheology and Microcirculation, DOI:10.3233/CH-131679, 2013.

[11] H. Hsiu, C.L. Hsu, M.Y. Jan. Relations between beat-to-beat microcirculatory blood flow and variations therein. Photomedicine and Laser Surgery, Vol.28, pp.785-792, 2010.

[12] H. Hsiu, S.M. Huang, T.L. Hsu TL. Evaluation of the function of arteriolar opening by variability in microcirculatory blood flow following angiotensin II administration in rats. Biorheology, Vol.47, pp.239-253, 2010.

[13] Y.Y. Wang Lin, T.L. Hsu, M.Y. Jan, W.K. Wang. Theory and applications of the harmonic analysis of arterial pressure pulse waves, Journal of Medical and Biological Engineering, Vol.30, pp.125-131, 2010.

[14] 14. Neki NS, Sing RB, Rastogi SS. How brain influences neuro-cardiovascular dysfunction, J Assoc Physicians India 2004;52:223-230.

[15] Task force of the European society of cardiology and the North American society of pacing and electrophysiology. Heart rate variability; Standards of measurement, physiological interpretation, and clinical use. Circulation, Vol.93, pp.1043-1065, 1996.

[16] R.B. Singh, G. Cornélissen, A. Weydahl, O. Schwartzkopff, G. Katinas, K. Otsuka, Y. Watanabe, S. Yano, H. Mori, Y. Ichimaru Y, G. Mitsutake, D. Pella, L. Fanghong, Z. Zhao, R.S. Rao, A. Gvozdjakova, F. Halberg. Circadian heart rate and blood pressure variability considered for research and patient care, Internal Journal of Cardiology, Vol.87, pp.9-28, 2003.

[17] Y.Y. Wang Lin, M.Y. Jan, C.S. Shyu, C.A. Chiang, W.K. Wang. The natural frequencies of the arterial system and their relation to the heart rate. IEEE Transaction on Biomedical Engineering, Vol.51, pp.193-195, 2004. 